\title{
PASSIFLORA TRYPHOSTEMMATOIDES AND ITS ALLIES
}

\author{
by \\ Lauritz B. Holm-Nielsen \\ Botanical Institute, University of Aarhus \\ Aarhus, Denmark
}

and

Peter M. Jørgensen

Instituto de Biologia

P. Universidad Catolica del Ecuador

Apartado 2184, Quito, Ecuador

\section{ABSTRACT}

The status of Passiflora subgenus Tryphostemmatoides (Harms) Killip is discussed and a key to the species of this group is provided.

\section{INTRODUCTION}

Killip (1938) separated the Subgenus Tryphostemmatoides (Harms) Killip from the closely related Subgenera Deidamioides (Harms) Killip and Plectostemma Mast. by a combination of tendril bearing inflorescences, non plicate operculum, and bracts crowded at base of the pedicel (for terminology see Killip 1938 and Jørgensen et al. 1984). In our oppinion all species of Subg. Tryphostemmatoides have at apex a slightly plicate or at the least rough operculum, just as seen in Passiflora filipes or $\mathrm{P}$. tenella, both recognized members of Subg. plectostemma. The floral morphology of Subg. Tryphostemmatoides does not deviate from the general arrangement in Plectostemma. Tryphostemmatoides is accordingly reduced to a Section of Subg. Plectostemma. 
Subg. Deidamioides and Apodogyne Killip are in our oppinion very likely to be included in Plectostemma as well, but no material has been studied and no decision is made.

The species P. gracillima and P. tryphostemmatoides were, as indicated by Knapp \& Malilet (1984), wrongly equated in Woodson \& Schery (1958). On the basis of this and the insufficient key characters in Killip's monograph (1938), plus the description of P. arbelaezii L.Uribe, we have felt that a key, short description and a distribution map for the Section were needed.

Passiflora Subg. Plectostemma Mast. emend P.M. Jørgensen \& Holm-Nielsen Sect. Tryphostemmatoides Harms.

Passiflora Sect. Tryphostemmatoides Harms, in Engl. \& Prantl, Nat. Pflanzenfam., ed.2. 21: 500. (1925).

Passiflora Subg. Tryphostemmatoides (Harms) Killip, P. Field. Mus. Nat. Hist., Bot. Ser. 19(1):24-25. (1938). Type species: Passiflora tryphostemmatoides Harms.

Delicate lianas, glabrous throughout. Stem terete or angular, striate. Stipules $1 \mathrm{~mm}$, setaceous, often deciduous. Petioles with 2-4 sessile or stipitate glands borne at the junction to blade. Inflorescences axillary, with 2 pedicellate flowers, terminating in an often deciduous, minute tendril. Bracteoles setaceous, often deciduous. Calyx patelliform. Corona 1- to 3-seriate. Operculum plicate to slightly plicate, or rough. Nectar ring annular. Limen cupuliform. Ovary glabrous. Seeds reticulate.

\section{Key to Passiflora Subg. Plectostemma Sect. Tryphostemmatoides}

1a. Limen less than $1.5 \mathrm{~mm}$ high. Corona uniseriate. Flower less than $1.7 \mathrm{~cm}$ wide. Fruit subglobose $1.8-2.7 \times 1.5-2.5 \mathrm{~cm}$. Fig. 1. ............. P. tryphostemmatoides

1b. Limen more than $1.5 \mathrm{~mm}$ high. Corona 1- to triseriate. Flowers more than $1.7 \mathrm{~cm}$ wide. Fruit ovoid or fusiform $2.5-6.4 \times 1.5-2.4 \mathrm{~cm}$.

2a. Limen closely apressed the androgynophore, margin entire. Corona 1or biseriate. Peduncle less than

$3.5 \mathrm{~cm}$ long. Fig. 1.......... gracillima

2b. Limen free, margin denticulate. Corona 2or triseriate. Peduncle longer than $3.5 \mathrm{~cm} \ldots \ldots \ldots \ldots \ldots \ldots \ldots \ldots \ldots \ldots \ldots$. F. arbelaezii 


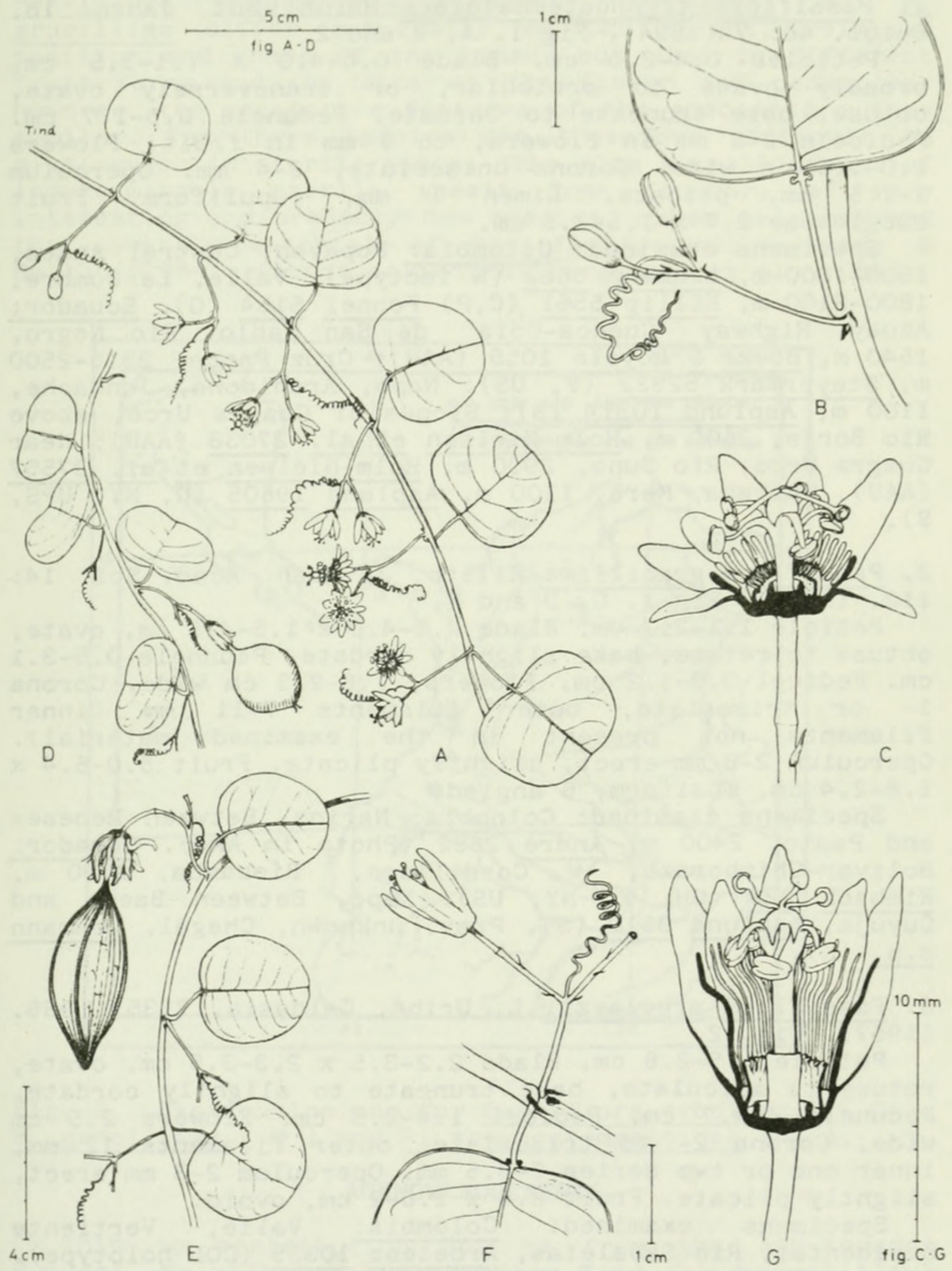

Fig. 1. Passiflora tryphostemmatoides A. fruit (Lehmann 5662), B. cross section of flower (Asplund 19605). P. gracilima (Rimbach 222) C. fruit, D. cross section of flower. 
1. Passiflora tryphostemmatoides Harms, Bot. Jahrb. 18, Beibl. 46: 7 (1894). Fig 1. A, B and 2 .

Petioles $0.4-2.0 \mathrm{~cm}$. Blade 0.6-4.0 $\mathrm{x} 1.1-3.5 \mathrm{~cm}$, broadly ovate to orbicular, or transversely ovate, obtuse, base truncate to cordate. Peduncle 0.5-1.7 cm. Pedicels 2-6 $\mathrm{mm}$ in flowers, to $9 \mathrm{~mm}$ in fruit. Flowers $1.0-1.6 \mathrm{~cm}$ wide. Corona uniseriate, 2-4 mm. Operculum 1-1.5 mm, plicate. Limen $1 \mathrm{~mm}$, cupuliform. Fruit subglobose $2.7 \times 1.5-2.5 \mathrm{~cm}$.

Specimens examined: Colombia: Popayan, Central Andes, 1500-1800 m, Lehmann 5662 (K isotype). Valle, La Cumbre, 1800-2100 m, Killip $5 \overline{561}$ (C,P) Pennel 5154 (G). Ecuador: Azuay, Highway Cuenca-Cola de San Pablo, Río Negro, $1540 \mathrm{~m}$, Boeke \& Loyola 1056 (AAU); Cruz Pamba, 2315-2500 $\mathrm{m}$, Steye $\overline{\mathrm{rmark}} 52 \overline{932}(\mathrm{~F}, \mathrm{US})$. Napo, Archidona, Jundache,

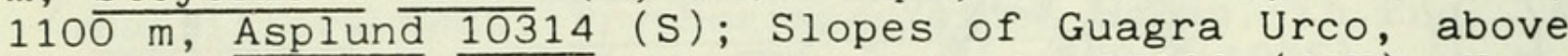
Rio Borja, $2600 \mathrm{~m}$, Holm-Nielsen et al. 27038 (AAU); Near Guagra Urco, Río Suno, $2900 \mathrm{~m}$, Holm-Nielsen et al. 27557

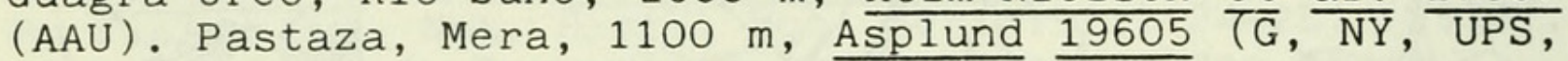
S) .

2. Passiflora gracillima Killip, J. Wash. Acad. Sci. 14: 112. (1924). Fig 1. C, D and 2 .

Petiole $1.1-2.7 \mathrm{~cm}$. Blade $2.0-4.0 \times 1.5-4.5 \mathrm{~cm}$, ovate, obtuse to retuse, base slightly cordate. Peduncle 0.5-3.1 $\mathrm{cm}$. Pedicel 0.8-1.2 cm. Flowers, $1.8-2.3 \mathrm{~cm}$ wide. Corona 1- or triseriate, outer filaments 7-11 mm (inner filaments not present in the examined material). Operculum 2-3 mm erect, slightly plicate. Fruit 5.0-6.4 x $1.8-2.4 \mathrm{~cm}$, fusiform, 6 angled.

Specimens examined: Colombia: Narino, Between Meneses and Pasto, $2400 \mathrm{~m}$, André 2882 (Photo in AAU). Ecuador: Bolivar-Chimborazo, W. Cordillera, Riobamba, $2600 \mathrm{~m}$, Rimbach 222 (GH, F, NY, US). Napo, Between Baeza and Cuyuja, Asplund 9610 (S). Prov. unknown, Chagal, Lehmann s.n. $(K)$.

3. Passiflora arbelaezii L. Uribe, Caldasia, 7(35): 335 . (1957). Fig. 2 .

Petiole $1.5-2.6 \mathrm{~cm}$. Blade $2.2-3.5 \times 2.3-3.5 \mathrm{~cm}$, ovate, retuse to apiculate, base truncate to slightly cordate. Peduncle 4-4.7 cm. Pedicel $1.4-2.5 \mathrm{~cm}$. Flowers $2.5 \mathrm{~cm}$ wide. Corona 2- to triseriate, outer filaments $12 \mathrm{~mm}$, inner one or two series $2-3.5 \mathrm{~mm}$. Operculum 2-3 $\mathrm{mm}$ erect, slightly plicate. Fruit $2.6 \times 1.6-2 \mathrm{~cm}$, ovoid.

Specimens examined: Colombia: Valle, Vertiente occidental, Río Sabaletas, Arbeleaz 10335 (COL holotype). Panama: Chiriqui, Hart 104 (K). 
$\frac{\text { Passiflora }}{\text { gracillima }} \frac{\text { tryphostemmatoides }}{\text { differ especially }}$ in the height, relative position and shape of the limen, but also in different fruit forms and the size of the flower and peduncles, however, the vegetative features of the species are very similar. The three species are therefore difficult to distinguish in sterile state, this plus a small amount of indeterminable sterile sheets from Ecuador, of very interesting and probably new species, makes us recommend that sterile material of Passiflora, unlike Knapp \& Mallet (1984), is not collected (Jørgensen et al. 1984).

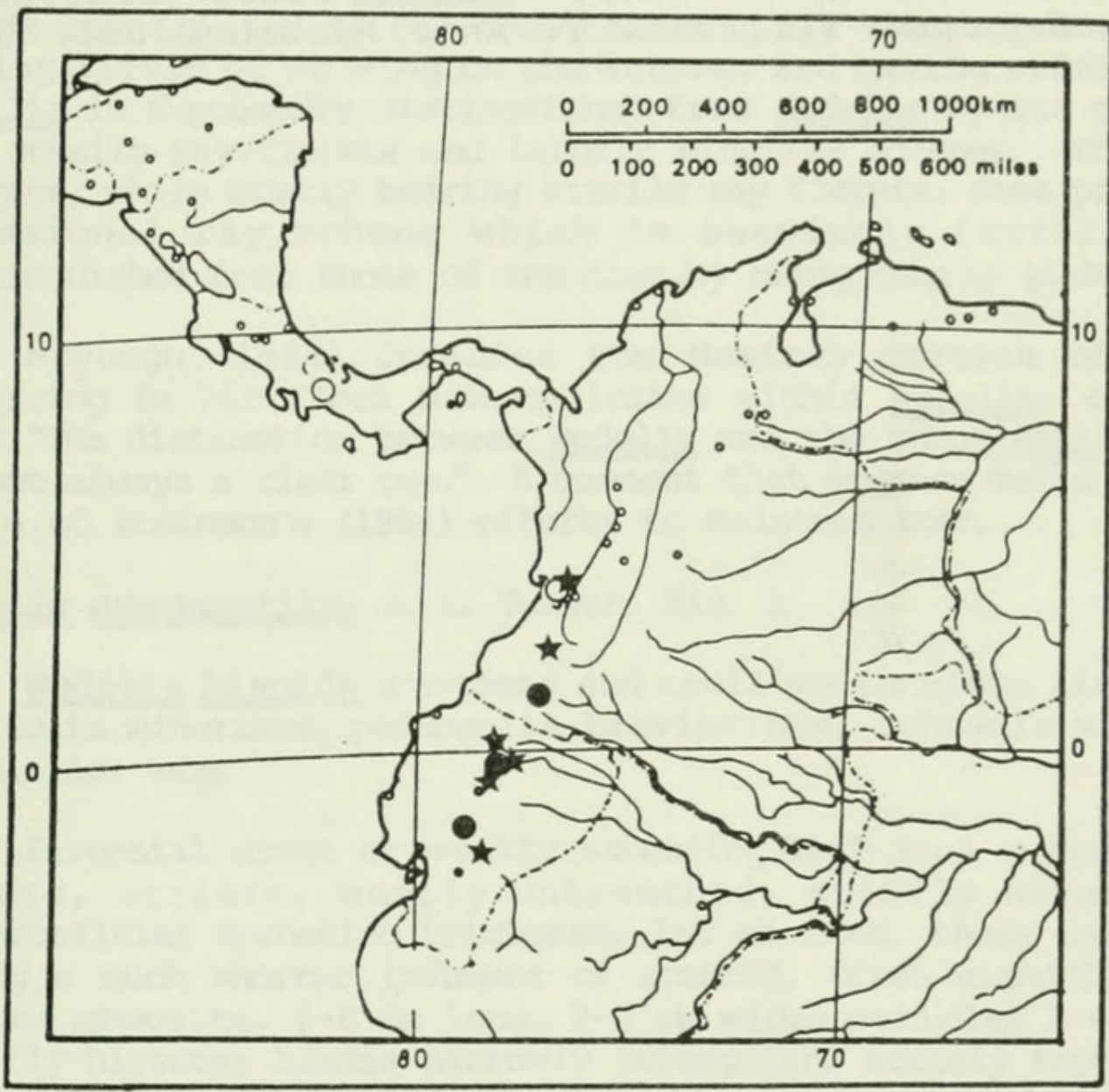

Fig. 2. Distibution map for Passiflora arbelaezii ( 0 ), $\underline{\text { P. gracillima }}(-)$ ) and $\underline{\text { P. tryphostemmatoides }}(\star)$.

\section{ACKNOWLEDGEMENTS}

This is contribution No. 54 from the AAU-Ecuador Project. We wish to thank Kirsten Tind for her skillful drawings. 


\section{REFERENCES}

Jørgensen, P.M., J.E. Lawesson \& L.B. Holm-Nielsen. 1984. A Guide to Collecting Passion Flowers. Ann. Missouri Bot. Gard. 71 (1172-1174).

Killip, E.P. 1938. The American Species of Passifloraceae. Publ. Field. Mus. Nat. Hist., Bot. Ser. 19: $1-613$.

Knap, S. \& J. Mallet. 1984. Two New Species of Passiflora (Passifloraceae) from Panama with Comments on their Natural History. - Ann. Missouri Bot. Gard. 71: 1068-1074.

Woodson, R.E. \& R.W. Schery. 1958. Passifloraceae in Flora of Panama VII Fasc. 1. Ann. Missouri Bot. Gard. 45: $1-22$. 


\section{$2 \mathrm{BHL}$ Biodiversity Heritage Library}

Holm-Nielsen, Lauritz Broder and Jørgensen, Peter. 1986. "Passiflora tryphostemmatoides and its allies." Phytologia 60, 119-124. https://doi.org/10.5962/bhl.part.3796.

View This Item Online: https://www.biodiversitylibrary.org/item/48955

DOI: https://doi.org/10.5962/bhl.part.3796

Permalink: https://www.biodiversitylibrary.org/partpdf/3796

\section{Holding Institution}

New York Botanical Garden, LuEsther T. Mertz Library

\section{Sponsored by}

The LuEsther T Mertz Library, the New York Botanical Garden

\section{Copyright \& Reuse}

Copyright Status: In copyright. Digitized with the permission of the rights holder.

Rights Holder: Phytologia

License: http://creativecommons.org/licenses/by-nc-sa/3.0/

Rights: https://biodiversitylibrary.org/permissions

This document was created from content at the Biodiversity Heritage Library, the world's largest open access digital library for biodiversity literature and archives. Visit BHL at https://www.biodiversitylibrary.org. 